\title{
A Spaceborne Wide-Area Surveillance Mode and Its Application in Maritime Monitoring
}

\author{
Shi-Chao Zheng, ${ }^{1,2}$ Hong-Jun Song, ${ }^{1}$ Robert Wang, ${ }^{1}$ Yun-Hua Luo, ${ }^{1,2}$ and Kun Wu ${ }^{1,2}$ \\ ${ }^{1}$ Institute of Electronics, Chinese Academy of Sciences, Beijing 100190, China \\ ${ }^{2}$ University of the Chinese Academy of Sciences, Beijing 100039, China \\ Correspondence should be addressed to Shi-Chao Zheng; jerryiszsc@163.com
}

Received 30 June 2013; Revised 10 September 2013; Accepted 5 October 2013

Academic Editor: Ananda Sanagavarapu Mohan

Copyright (c) 2013 Shi-Chao Zheng et al. This is an open access article distributed under the Creative Commons Attribution License, which permits unrestricted use, distribution, and reproduction in any medium, provided the original work is properly cited.

\begin{abstract}
The spaceborne wide-area surveillance mode, achieved by a modified TOPSAR mode, is proposed for maritime monitoring. In the proposed mode, the requirements of detecting targets from different aspect angles with a high revisit rate can be satisfied by modifying the TOPSAR mode which steers antenna in the azimuth direction. The feasibility as well as its application in maritime monitoring are validated by theoretical analysis and simulation experiments.
\end{abstract}

\section{Introduction}

One important task of the radar system is to monitor the moving targets on the wide area at any time and in all weather conditions. In order to surveil a wider area with respect to conventional SAR/GMTI mode, the airborne widearea surveillance mode has been developed and implemented in many real systems, such as phased array multifunctional imaging radar (PAMIR) [1-5] and joint surveillance target attack radar system (JointSTARS) [6].

Spaceborne SAR takes the advantage of large coverage and therefore promises a bright application future in maritime monitoring [7]. However, some system requirements should be met to guarantee efficient maritime monitoring. First, the revisit rate has to be high enough to permit a meaningful tracking of the detected targets. In addition, the ability to detect the same target from different aspect angles is also needed. In this paper, these requirements can be achieved by modifying the TOPSAR mode which also steers antenna in the azimuth direction [8-11], refered to as spaceborne wide-area surveillance mode. In the spaceborne wide-area surveillance mode, by steering antenna in the azimuth direction, multiple images of the same scene are obtained. Therefore, targets can be detected from different aspect angles and successfully tracked.
Since the coverage of the spaceborne wide-area surveillance mode is much larger than that of the airborne widearea surveillance mode, maritime targets on broad sea area can be surveilled in a shorter time. What is more, as the radar cross section of targets and the signal-to-noise ratio (SNR) vary with different incidence angles, the capability of target detection can be improved because the targets are illuminated from different aspect angles in the spaceborne wide-area surveillance mode. In addition, the tracking of targets can also be utilized to reduce the false alarm probability.

The paper is organized as follows. Section 2 introduces the implementation of the spaceborne wide-area surveillance mode, and the system analysis of the spaceborne wide-area surveillance mode is also presented. Section 3 shows the procedure of maritime monitoring in the spaceborne widearea surveillance mode. In Section 4, the proposed idea is validated by simulation. Finally, Section 5 concludes the whole paper.

\section{Implementation and System Analysis of the Spaceborne Wide-Area Surveillance Mode}

In this section, we introduce the airborne wide-area surveillance mode and then extend it to the spaceborne case for 
maritime monitoring. The system analysis of the spaceborne wide-area surveillance mode is also presented.

\subsection{Implementation of the Spaceborne Wide-Area Surveillance} Mode. The airborne wide-area surveillance (WAS) mode is an effective technique to monitor wide areas using a narrow antenna beam in a scanning operation. This is achieved by steering the antenna in the azimuth direction in a cyclic way after a certain time span. Thus, different parts of the ground are scanned successively, and then a wide area can be monitored. Such a scan operation can be fulfilled efficiently with a phased-array antenna [1-5].

Although airborne wide-area surveillance mode performs well in ground traffic monitoring and battlefield surveillance, the extension to the surveillance of broader area is restricted because of its limited coverage. Therefore, the support of surveillance provided by satellites which are more able than aircrafts to cover widely dispersed areas is firstly taken into consideration to realize maritime monitoring in the paper.

In this paper, the case of revisiting the same area by antenna steering in modified TOPSAR mode is referred to as the spaceborne wide-area surveillance mode. Two manners can be considered to realize the revisit of the same area. In one manner, the beam illuminates the same area several times during a burst, and then the beam switches to another subswath; afterwards, the procedure repeats cyclically. In the other manner, the beam covers all the subswaths first, and then it revisits the same area by means of steering the antenna in azimuth (as shown in Figure 1). Since the fundamentals of the two manners are similar, the following analysis is only based on the second one.

In the proposed spaceborne wide-area surveillance mode, the requirements discussed in Section 1 can be fulfilled. In addition, compared to airborne wide-area surveillance mode, the data obtained in spaceborne wide-area surveillance mode are focused in azimuth. Fine resolution images can be obtained, and because of the high SCR of sea area SAR images, CFAR detection can be utilized for maritime target detection [12]. Therefore, single-channel systems can be used for maritime monitoring in the spaceborne wide-area surveillance mode with lower cost and simpler configuration compared to multichannel systems.

2.2. The System Analysis of the Proposed Mode. In this section, the system analysis is presented: first, the timeline of the proposed mode is analyzed; second, the system performance including the effect of potential grating lobes and the target detectability of the proposed mode is discussed briefly.

The basic system parameters and requirements used in the system analysis are shown in Table 1.

2.2.1. The Timeline of the Proposed Mode. Before the analysis of the timeline, the PRF selection, which is critical to optimize the system performance, is presented. The choice is constrained by nadir returns and transmit events, to be avoided within the sampling window time. These constraints are shown in the diamond diagram in Figure 2. It should be

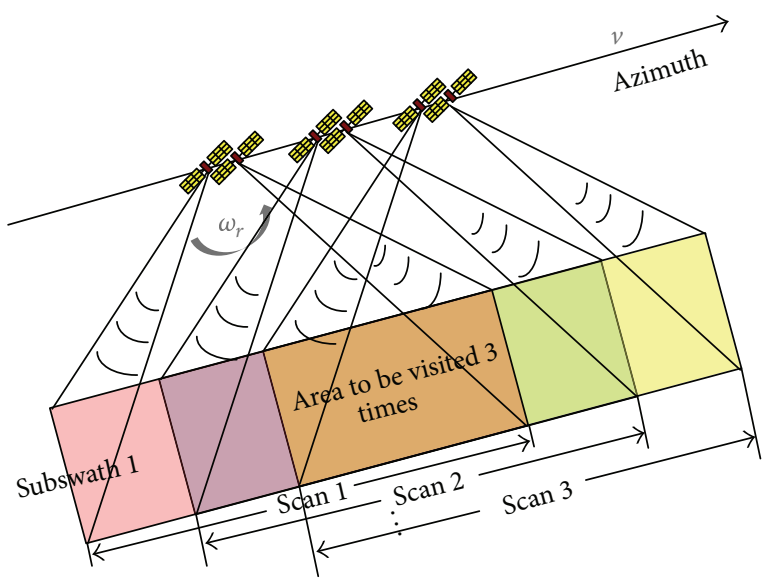

FIGURE 1: Scanning in one of the subswaths in spaceborne WAS mode with three revisits.

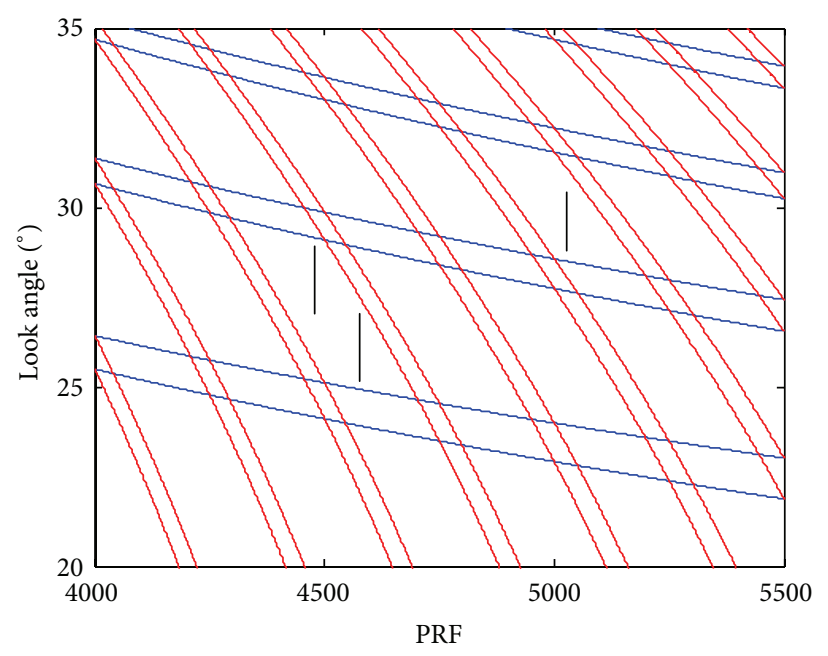

FIGURE 2: Diamond diagram showing the setting of PRF.

TABLE 1: The basic system parameters and the system requirements.

\begin{tabular}{lc}
\hline Parameter & Value \\
\hline Wavelength $(\mathrm{cm})$ & 3.125 \\
Platform velocity $(\mathrm{m} / \mathrm{s})$ & 7558 \\
Antenna length $(\mathrm{m})$ & 4 \\
Height $(\mathrm{km})$ & 600 \\
Azimuth resolution $(\mathrm{m})$ & 20 \\
Range resolution $(\mathrm{m})$ & 10 \\
Number of antenna columns & 32 \\
Transmit peak power $(\mathrm{W})$ & 3200 \\
Receiver noise level $(\mathrm{dB})$ & 4 \\
Swath requirement $(\mathrm{km})$ & $>100$ \\
Number of subswaths & 3 \\
Illumination repetition times & 3 \\
\hline
\end{tabular}

noticed that, in PRF designing, margins have to be assigned due to changes of the acquisition geometry, depending on the size of each swath. 


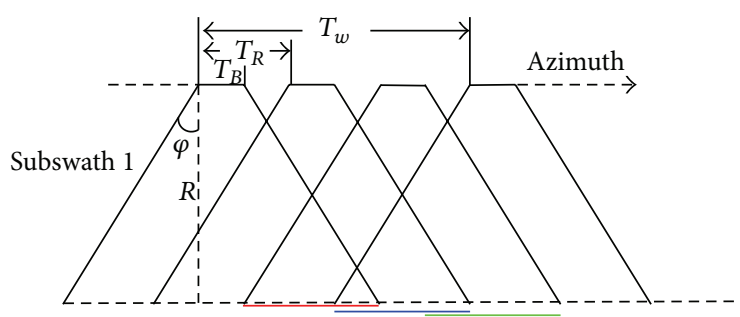

FIGURE 3: One subswath of spaceborne WAS mode with three revisits.

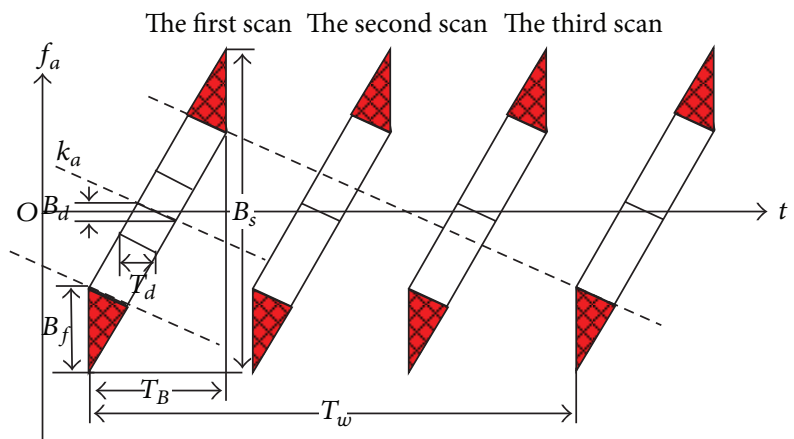

Figure 4: TFD properties of spaceborne WAS mode.

To ensure that the strip coverage that can be revisited $M$ times is continuous (as shown in Figure 3, the areas colored by red, blue, and green should be continuous), the distance that the platform moves during the cycle time, $T_{R}$, should be smaller than the effective size of the imaged area that can be revisited $M$ times in one subswath:

$$
(2 \varphi-\theta) R+V_{s} T_{B}-(M-1) V_{s} T_{R} \geq V_{s} T_{R},
$$

where $\varphi$ is the steering angle, $\theta$ is the $3 \mathrm{~dB}$ beam width in azimuth, $V_{s}$ is the satellite velocity, $T_{B}$ is the burst duration, and $T_{R}$ is defined as

$$
T_{R}=\sum_{i=1}^{N}\left(T_{B i}+T_{g}\right),
$$

where $N$ is the number of the subswaths, $T_{B i}$ is the burst duration of the $i$ th subswath, and $T_{g}$ is the switching time between different subswaths.

Based on the previous analysis, the spaceborne WAS acquisition in slow-time/frequency domain (TFD) is represented in Figure 4.

From the TFD of the proposed mode, it can be seen that the continuous strip coverage is achieved by imposing that the last target processed in the $n$th cycle is the first one in the main AAP beam in the $n+1$ th cycle (Figure 4 is based on the case of three revisits).

Afterwards, in order to simplify the analysis, the switching time is ignored, and the preliminary burst length can be calculated by

$$
T_{B}=\frac{(2 \phi-\theta) R}{(M N-1) V_{s}} .
$$

The rotation rate and the shrinking factor are defined as

$$
\begin{gathered}
\omega_{r}=\frac{2 \phi}{T_{B}}, \\
A=1+\frac{\omega_{r} R}{V_{g}} .
\end{gathered}
$$

Therefore, the azimuth resolution can be calculated by

$$
\rho_{a}=\frac{V_{s}}{B_{f}} \cdot A=\frac{\omega_{r} R+V_{s}}{B_{f}},
$$

where $B_{f}$ is the Doppler bandwidth of azimuth beam. Afterwards, the burst length should be adjusted based on the geometry difference of different subswaths to ensure the azimuth resolution of different subswaths to be the same. Because the burst length varies slightly between different subswaths and consequently the variance has little impact on the system analysis, the burst adjustment is not presented here.

2.2.2. The Influence of Grating Lobes. The implementation of a spaceborne WAS scheme requires to steer the antenna azimuth pattern (AAP) in the azimuth direction, and the sweeping is implemented by an electronic steering. In fact, the update rate demands for a phased array to be formed with a discrete number of rows/columns. The beam forming introduces grating lobes and antenna gain loss that become relevant at the large steering angles, which impacts on AASR and NESZ significantly.

In Figure 5, the AAP, the gain loss, the AASR, and the NESZ as functions of steering angle are plotted. It can be seen that the performance upgrades together with the increase of antenna columns. Therefore, an antenna with more columns can be utilized to suppress the influence of grating lobes.

2.2.3. The Analysis of Target Detectability. In the application of maritime target detection, the ambiguous signals may lead to false alarms, which will degrade the detectability of targets. For the spaceborne WAS mode, large steering angle, introducing grating lobes, makes the impact of ambiguous signal more severe (the worst case is shown in Figure 6(a)). In order to alleviate the problem, besides selecting an antenna with more columns, PRF should be properly selected to ensure that most of the grating lobe energy is folded outside the processed bandwidth (the best case is shown in Figure 6(b)) [13].

Based on the theory of phased-array antenna and the principle of azimuth ambiguity, the location scopes of the ambiguous signals and grating lobes can be expressed by (6) and (7), respectively:

$$
\begin{aligned}
& \operatorname{asin}\left(\frac{(m \cdot \mathrm{PRF}+2 \cdot V \cdot \sin \theta / \lambda)}{2 \cdot V} \cdot \lambda\right)-\frac{\theta_{3 d B}}{2} \\
& \quad \leq \theta_{m} \leq \operatorname{asin}\left(\frac{\left(m \cdot \mathrm{PRF}+2 \cdot V \cdot \frac{\sin \theta}{\lambda}\right)}{2 \cdot V} \cdot \lambda\right)+\frac{\theta_{3 d B}}{2},
\end{aligned}
$$




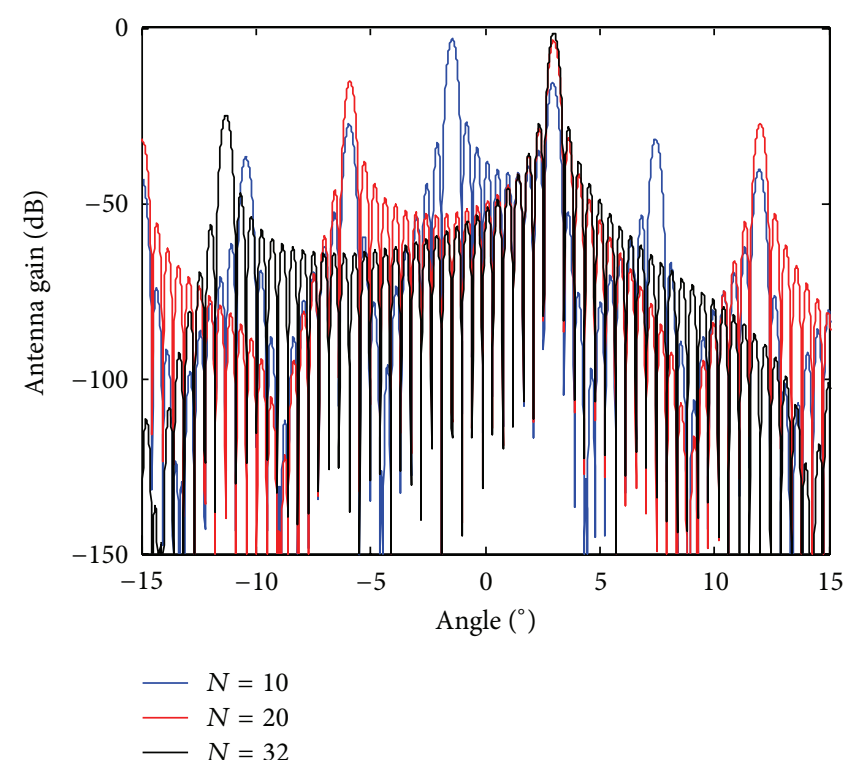

(a)

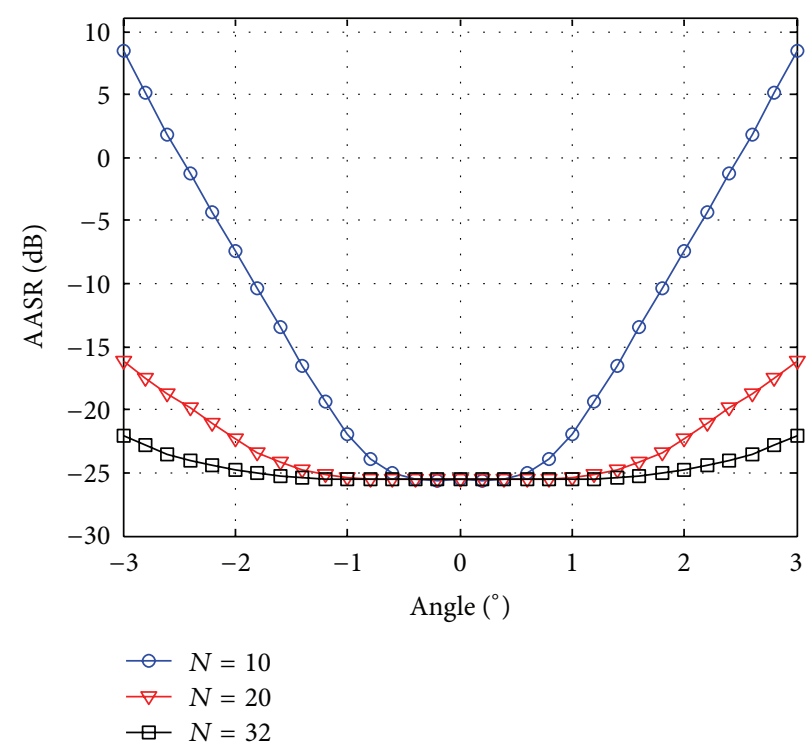

(c)

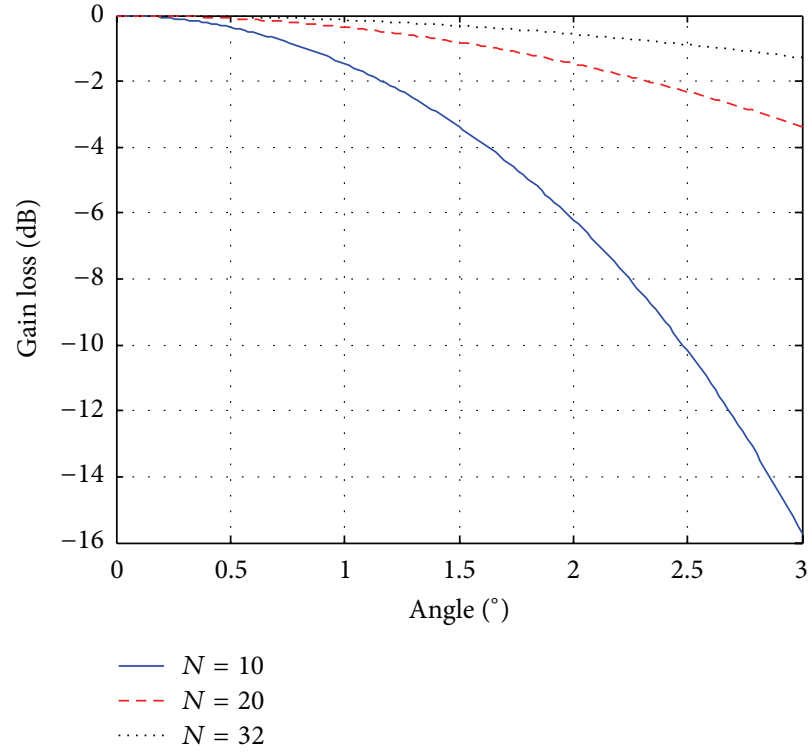

(b)

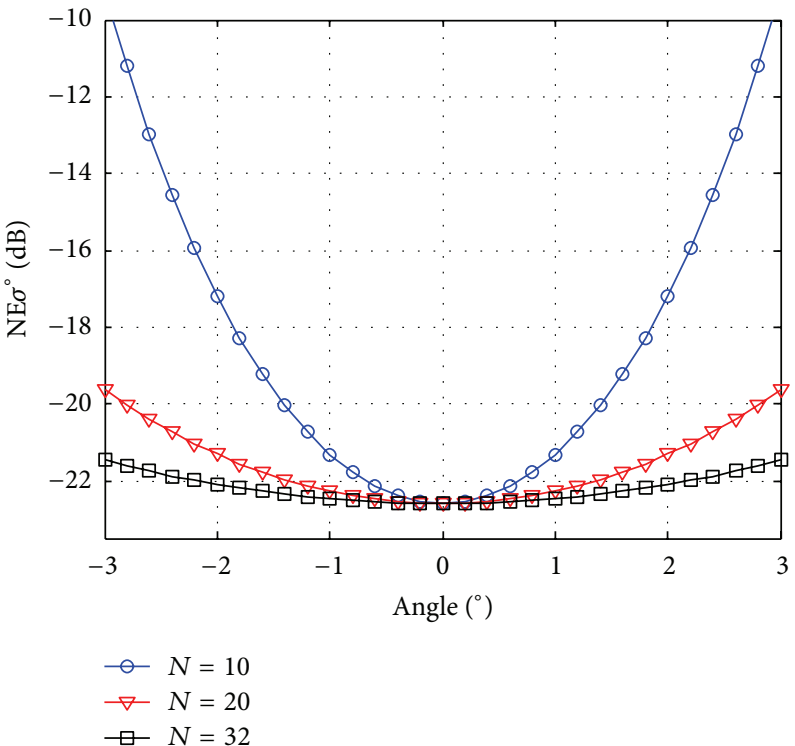

(d)

Figure 5: (a) AAP, assuming a steering to $3^{\circ}$, (b) Antenna gain loss, (c) AASR, and (d) NESZ for different number of antenna columns.

where $V$ is the equivalent velocity, $\theta$ represents the steering angle, $\lambda$ is the wavelength of the signal, $\theta_{3 d B}$ accounts for the antenna beam width in azimuth, and $m$ is the number of ambiguous signals,

$$
\begin{aligned}
& \operatorname{asin}\left(\frac{\lambda}{d} \cdot n+\sin \theta\right)-\frac{\theta_{3 d B}}{2} \\
& \leq \theta_{n} \leq \operatorname{asin}\left(\frac{\lambda}{d} \cdot n+\sin \theta\right) \\
&+ \frac{\theta_{3 d B}}{2}
\end{aligned}
$$

where $d$ is the space of antenna columns and $n$ is the number of grating lobes.

Therefore, the PRF should be optimized to avoid the overlapping of $\theta_{m}$ and $\theta_{n}$ to alleviate the impact of grating lobes.

In addition, the minimum detectable target radar cross section, which is impacted by the transmit power and the receiver noise level, should be calculated to analyze the detectability of targets. The calculation is based on the radar equation:

$$
\operatorname{SNR}=\frac{P_{t} G^{t} \sigma A_{e}}{\left(4 \pi R^{2}\right)^{2} \cdot k \cdot F \cdot T_{s} \cdot B_{n}}
$$




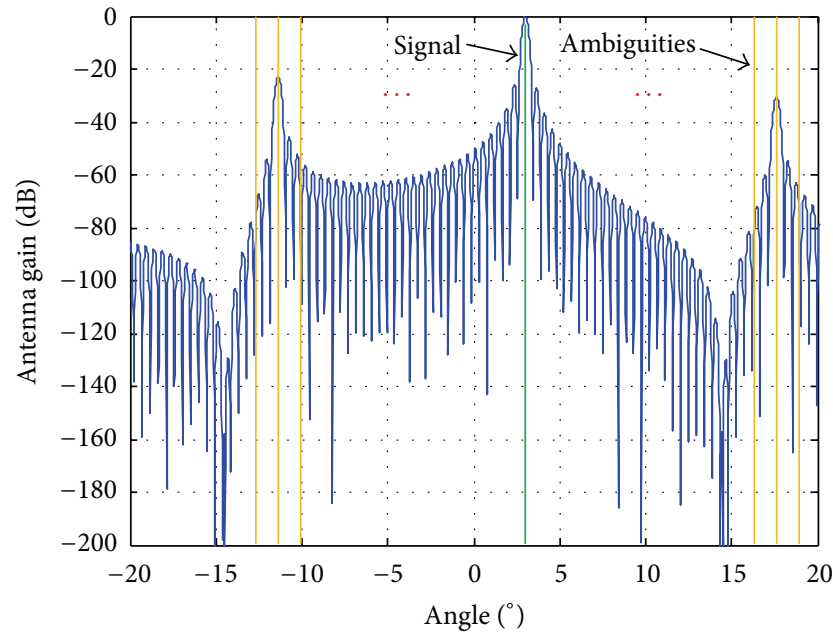

(a)

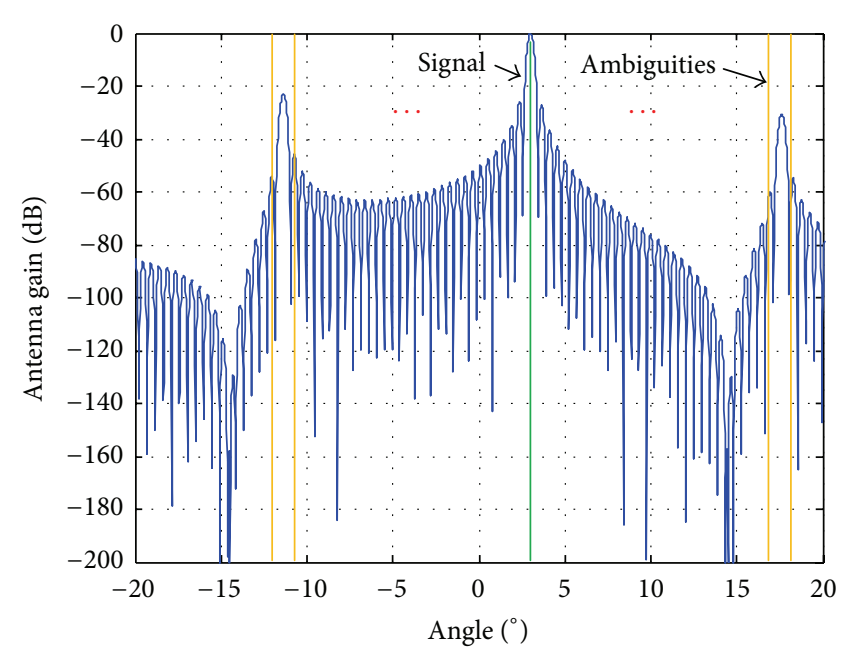

(b)

FIGURE 6: The impact of grating lobes with different PRF.

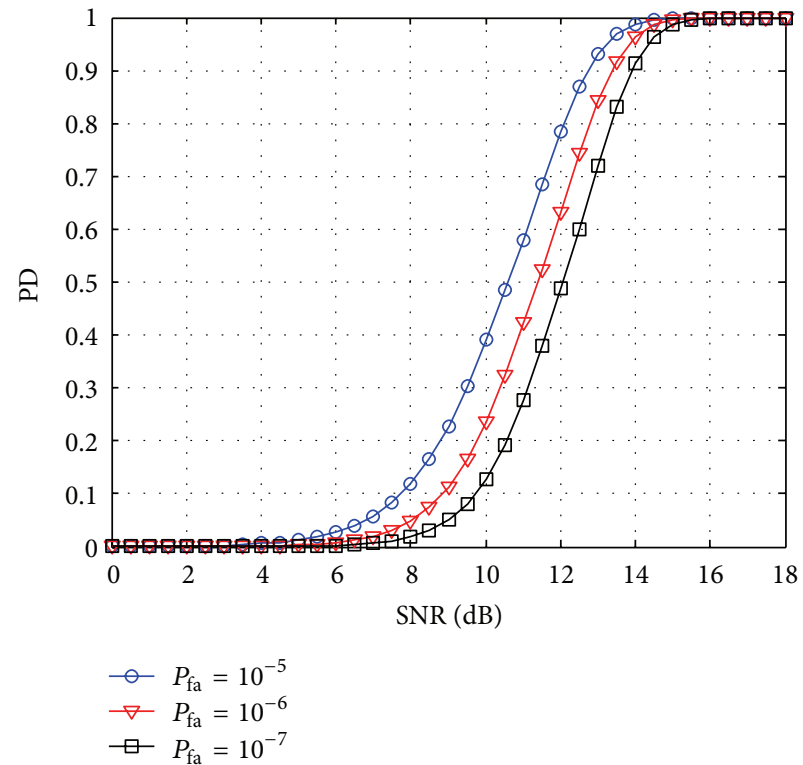

(a)

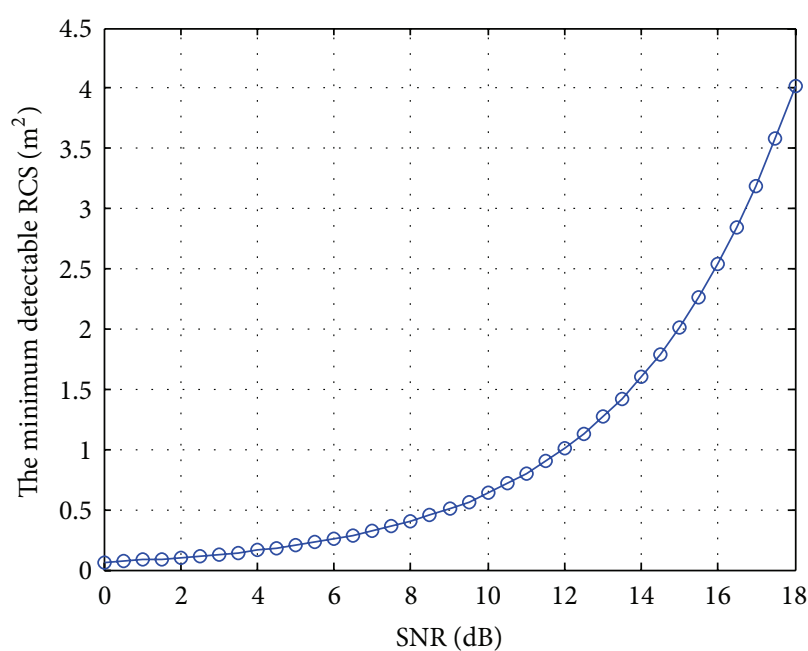

(b)

FIGURE 7: The detection probability (a) and minimum detectable radar cross section (b) as functions of SNR.

where $P_{t}$ is the transmit power of the antenna, $G^{t}$ represents the antenna gain, $\sigma$ is the radar cross section of the target, $A_{e}$ accounts for the effective antenna aperture, $R$ is the range between the radar and the target, $k$ is Boltzmann's constant, $F$ represents the receiver noise figure, $T_{s}$ is the total effective system noise temperature, and $B_{n}$ accounts for the receiver bandwidth.

It can be seen from (8) that SNR, which has a large effect on the probability of detection, improves with higher transmit power or lower receiver noise level. The detection probability and the minimum detectable radar cross section as functions of SNR are plotted in Figure 7, where the improvement of the target detectability with the increase of SNR is shown.

\section{The Application in Maritime Monitoring}

Since spaceborne SAR is prevailing to airborne SAR in the aspects of coverage, the spaceborne wide-area surveillance mode has a great advantage in maritime monitoring. Under this mode the same area can be revisited several times by antenna steering; then targets can be detected from different aspect angles and successfully tracked. As fine resolution 


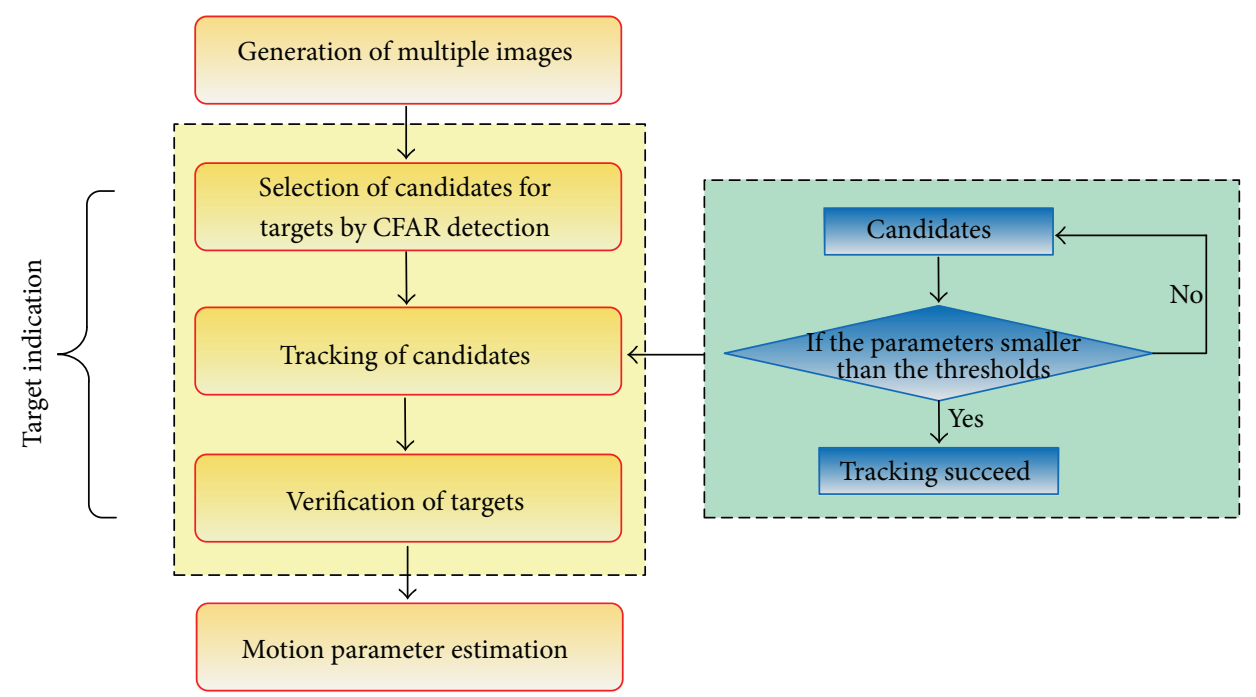

FIGURE 8: The procedure of maritime monitoring.

images can also be obtained, the candidates for maritime targets can be selected by CFAR detection, and the targets are verified by the tracking of candidates. Finally, the motion parameters of maritime targets are obtained by estimating their displacement vectors.

The motion parameter estimation will be done for each target individually. As no target has been detected so far, target candidates are selected by a CFAR processor first.

As is shown in Figure 8, after CFAR detection, the candidates of successive images are tracked. If the tracking succeeds, the candidate is considered to be a target. Here, three parameters are used in the tracking: the difference of the numbers of pixels belonging to corresponding candidates, the angle between the principal axis direction and these two candidates of connection, and the displacement between the corresponding candidates in successive images (as shown in Figure 9). If all the three parameters are smaller than the thresholds, the corresponding candidates are considered to be one target.

Here, the block matching algorithm is used to obtain the displacement of parts of the image between successive images $s_{1}$ and $s_{2}$, which are called frames in this section [14]. To obtain the displacement vector, a window is positioned in the frame $s_{2}$, so that the candidate lies in its center. Then another window is located on the same position in frame $s_{1}$. The aim is to find the best match between the two windows by shifting the window in frame $s_{1}$ on every position within a neighborhood. For this purpose, the normalized cross correlation function (NCCF) criterion defined by (6) is used:

$$
\begin{aligned}
C_{N s_{2} s_{1}} & (\Delta x, \Delta y) \\
= & \frac{\sum_{x, y}\left[s_{2}(x, y) \cdot s_{1}(x-\Delta x, y-\Delta y)\right]}{\sqrt{\sum_{x, y} s_{2}^{2}(x, y) \cdot \sum_{x, y} s_{1}^{2}(x-\Delta x, y-\Delta y)}},
\end{aligned}
$$

where $(x, y)$ is one of the positions inside the window and $\Delta x$ and $\Delta y$ are the position shifts between the two windows.

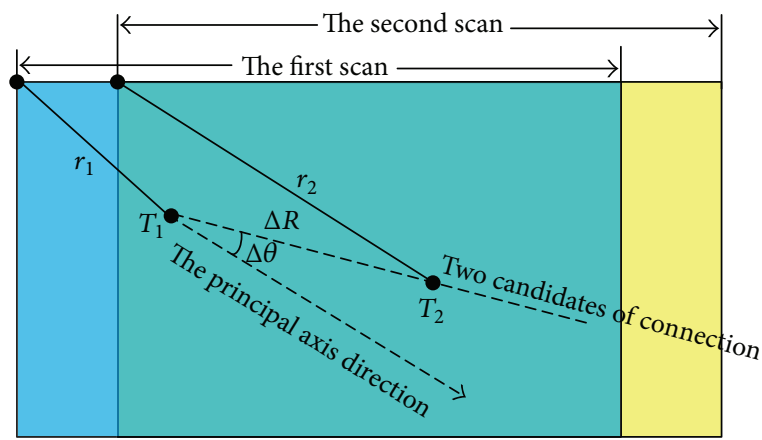

FIGURE 9: The tracking of candidates.

The motion vector of the selected candidate is then given by the shifts $\Delta x$ and $\Delta y$ which maximize the $C_{\mathrm{Ns}_{2} s_{1}}$.

Finally, the motion parameters of the targets that have been successfully tracked can be estimated by their displacements between successive images of the same area.

Since every target occupies several pixels, the estimation of motion parameters is different from that of point target. The block matching algorithm is utilized to make a coarse estimation first. Then the extracted targets are interpolated to obtain more accurate estimation results, and the NCFF criterion in one direction is used in the range direction and the azimuth direction, respectively.

Afterwards, the weighted average method that can get an accurate and robust estimation is utilized for the motion parameter calculation:

$$
v_{\mathrm{est}}=\frac{1}{\sum_{k} \alpha_{k}} \cdot \sum_{k}\left(\alpha_{k} \cdot v_{k}\right) \text {, }
$$

where $v_{k}$ and $\alpha_{k}$ are the estimated velocity and the weight of the $k$ th range bin (or the range line) that the target locates in, respectively. 


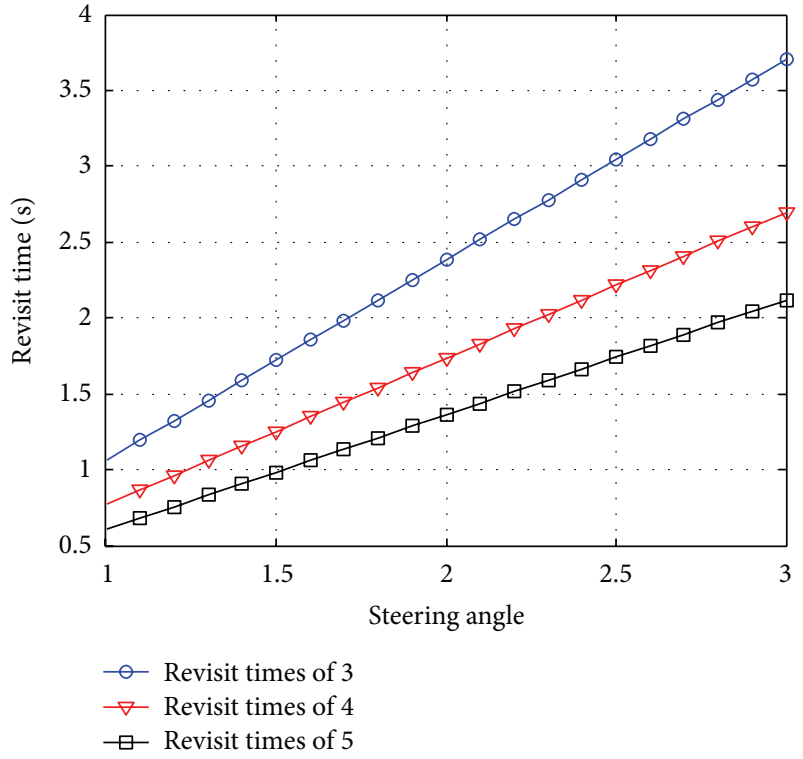

(a)

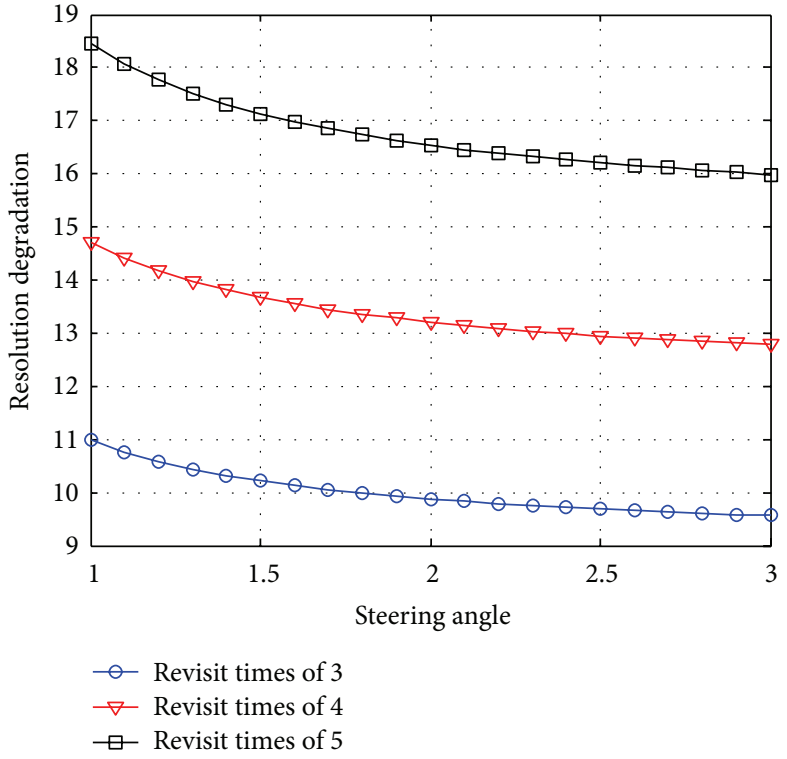

(b)

FIGURE 10: The revisit time (a) and the resolution degradation (b) with different revisit times.

In the spaceborne wide-area surveillance mode, multiple images of the same area are utilized. Although the collection of multiple images reduces the azimuth resolution, the target detection capability of each image does not degrade a lot because large targets are considered in this paper. Simultaneously, compared to conventional burst modes, the proposed mode based on TOPSAR can obtain better image quality with small scalloping effect. From another aspect, the capability of detecting the same target from different aspect angles and target tracking improves the detection performance. Actually, target tracking is also an achievement of the proposed maritime monitoring method which is not only utilized to reduce the false alarm probability.

\section{Simulation Experiments}

4.1. Feasibility Analysis. In this part, simulations are presented to validate that the system performance of spaceborne wide-area surveillance mode is acceptable without rigorous hardware requirements. The basic system parameters and requirements used in the simulations have been given in Table 1. In order to achieve the proposed requirements, the system parameters are selected based on the formulas given in Section 2. The results are listed in Table 2.

The revisit time and the azimuth resolution degradation (since these two parameters of different subswaths vary a little, only the result of one subswath is shown) obtained with different steering angles in the critical case are shown in Figure 10.

It can be found that the performance, such as the azimuth resolution, degrades as the revisit times or the number of subswaths increases. But more subswaths are required to obtain broader coverage; meanwhile, more revisit times are needed to track the targets. The analysis above shows that an
TABLE 2: Swath-dependent parameters and timeline optimized for the proposed mode.

\begin{tabular}{lcccc}
\hline Parameter & Symbol & IW1 & IW2 & IW3 \\
\hline PRF & & 4570 & 4470 & 5025 \\
Look angle $\left({ }^{\circ}\right)$ & & 26 & 28.3 & 30.6 \\
Slant range $(\mathrm{km})$ & $R$ & 667.5612 & 681.4485 & 697.0730 \\
Burst length & $T_{B}$ & 1.1819 & 1.2070 & 1.2354 \\
Echoes $/$ burst & & 5401 & 5396 & 6208 \\
AAP sweep rate $\left({ }^{\circ} / \mathrm{s}\right)$ & $\omega_{r}$ & 0.0886 & 0.0868 & 0.0848 \\
\hline
\end{tabular}

antenna with more columns can be utilized to upgrade the performances, which causes the increase of the cost and the power consumption. Thus, the selection of system parameters in spaceborne WAS mode must be compromised.

From the simulation results, it can be seen that the system requirements are possible to be achieved in practice, and the performance with corresponding system parameters, such as the minimum detectable target radar cross section, is also acceptable. Therefore, the proposed spaceborne wide-area surveillance mode is feasible and reasonable.

\subsection{The Simulation of the Application in Maritime Monitoring.} In the simulation, three targets with different velocities are set and other parameters are the same with the simulation above. The simulation flowchart is shown in Figure 11. Figures 11(a) and 11(b) show the images of successive visits; Figures 11(c) and $11(\mathrm{~d})$ are the results after CFAR detection. In order to avoid splits of the single target, dilation is used. Afterwards, the targets are tracked to reduce false alarm probability, and false alarms exist in both the results of successive visits after CFAR detection and dilation are rid through tracking 
TABLE 3: Motion estimation results.

\begin{tabular}{lcccr}
\hline & \multicolumn{2}{c}{ Azimuth velocity } & \multicolumn{2}{c}{ Range velocity } \\
& Actual value $(\mathrm{m} / \mathrm{s})$ & Estimated value $(\mathrm{m} / \mathrm{s})$ & Actual value $(\mathrm{m} / \mathrm{s})$ & 5 \\
Estimated value $(\mathrm{m} / \mathrm{s})$ \\
\hline Target 1 & -15 & -14.89 & 6 & 5.30 \\
Target 2 & 10 & 9.63 & 3 & 6.25 \\
Target 3 & 5 & 5.24 & 3.15 \\
\hline
\end{tabular}

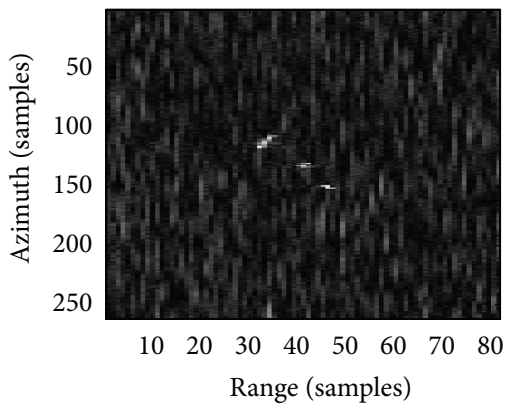

(a)

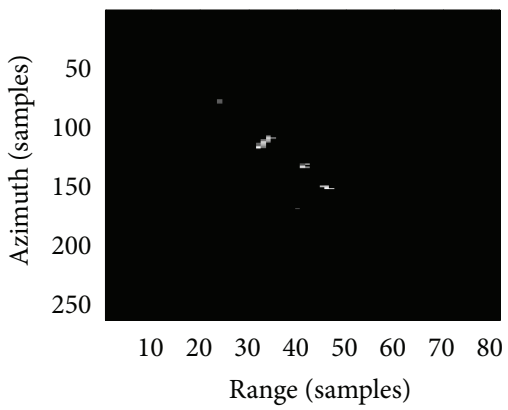

(d)

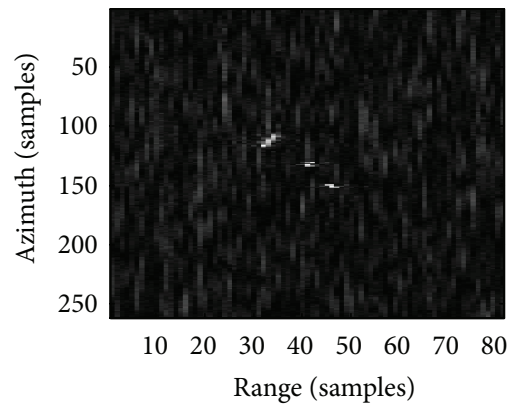

(b)

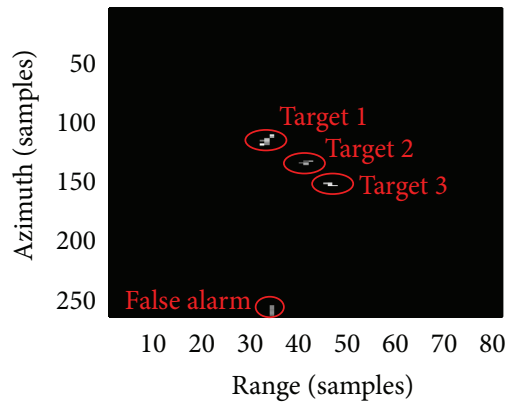

(e)

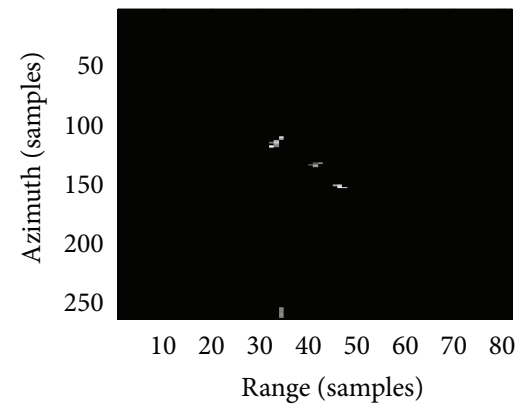

(c)

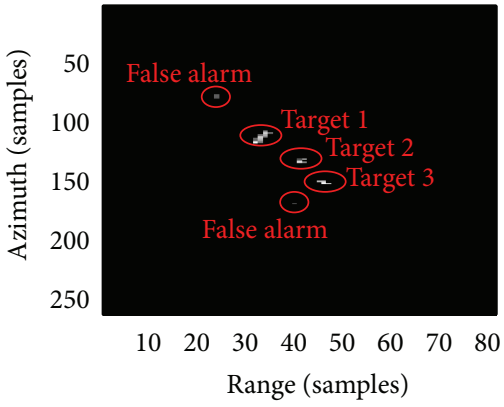

(f)

FIgURE 11: The simulation flowchart.

process; the results are shown in Figures 11(e) and 11(f). Finally, motion parameters are estimated.

As described in Section 3, the block matching algorithm and the weighted average method are utilized to estimate the motion parameters. Because the motion parameters are estimated in the image domain, the accuracy becomes higher as the image resolution improves. In addition, the calculation accuracy can be improved by the interpolation after target extraction since the coherence of corresponding targets in successive images is strong. In this simulation, the motion parameters of the three targets are estimated after eight times interpolation, and the results are given in Table 3.

\section{Conclusion}

In this paper, wide area surveillance from space has been realized based on modified TOPSAR, referred to as spaceborne wide-area surveillance mode. Its application in maritime monitoring is also discussed. The capability of maritime target detection can be improved because the targets are illuminated from different aspect angles in spaceborne widearea surveillance mode, and the tracking of targets is utilized to reduce the false alarm probability, and it also improves the performance. To verify the theoretical conclusions and demonstrate the efficiency of the proposed idea, simulations have been carried out. The results show that the spaceborne wide-area surveillance mode is feasible, and it has a big application potential in maritime monitoring.

\section{References}

[1] D. Cerutti-Maori, J. Klare, A. R. Brenner, and J. H. G. Ender, "Wide-area traffic monitoring with the SAR/GMTI system PAMIR," IEEE Transactions on Geoscience and Remote Sensing, vol. 46, no. 10, pp. 3019-3030, 2008.

[2] H. Yan, F. Li, R. Wang, M. J. Zheng, C. G. Gao, and Y. K. Deng :, "Moving targets extraction in multichannel widearea surveillance system by exploit sparse phase matrix," IEE Proceedings Radar, Sonar and Navigation, no. 9, pp. 913-920, 2012.

[3] D. Cerutti-Maori, W. Bürger, J. H. G. Ender, and A. R. Brenner, "Experimental results of ground moving target detection achieved with the multichannel SAR/MTI system PAMIR," Proceedings of the European Microwave Association, no. 2, pp. 122-127, 2006.

[4] J. H. G. Ender, P. Berens, A. R. Brenner, L. Rößing, and U. Skupin, "Multi channel SAR/MTI system development 
at FGAN: from AER to PAMIR," in Proceedings of the IEEE International Geoscience and Remote Sensing Symposium (IGARSS '02), pp. 1697-1701, Toronto, Canada, June 2002.

[5] J. H. G. Ender and A. R. Brenner, "PAMIR-a wideband phased array SAR/MTI system," Proceeding of the IEE Radar, Sonar and Navigation, vol. 150, no. 3, pp. 165-172, 2003.

[6] J. N. Entzminger Jr., C. A. Fowler, and W. J. Kenneally, "JointSTARS and GMTI: past, present and future," IEEE Transactions on Aerospace and Electronic Systems, vol. 35, no. 2, pp. 748-761, 1999.

[7] K. Ouchi, S. Tamaki, H. Yaguchi, and M. Iehara, "Ship detection based on coherence images derived from cross correlation of multilook SAR images," IEEE Geoscience and Remote Sensing Letters, vol. 1, no. 3, pp. 184-187, 2004.

[8] F. De Zan and A. M. Guarnieri, "TOPSAR: terrain observation by progressive scans," IEEE Transactions on Geoscience and Remote Sensing, vol. 44, no. 9, pp. 2352-2360, 2006.

[9] A. Meta, J. Mittermayer, P. Prats, R. Scheiber, and U. Steinbrecher, "TOPS imaging with TerraSAR-X: Mode design and performance analysis," IEEE Transactions on Geoscience and Remote Sensing, vol. 48, no. 2, pp. 759-769, 2010.

[10] W. Xu, P. Huang, Y. Deng, J. Sun, and X. Shang, "An efficient approach with scaling factors for TOPS-Mode SAR data focusing," IEEE Geoscience and Remote Sensing Letters, vol. 8, no. 5, pp. 929-933, 2011.

[11] W. Xu, Study on spaceborne terrain observation by progressive scans SAR (TOPSAR) mode. [Ph.D. dissertation], Institute of Electronics, Chinese Academy of Sciences, Beijing, China, 2011.

[12] K. Eldhuset, "An automatic ship and ship wake detection system for spaceborne sar images in coastal regions," IEEE Transactions on Geoscience and Remote Sensing, vol. 34, no. 4, pp. 1010-1019, 1996.

[13] D. D’Aria, F. De Zan, D. Giudici, A. M. Guarnieri, and F. Rocca, "Burst-mode SARs for wide-swath surveys," Canadian Journal of Remote Sensing, vol. 33, no. 1-4, pp. 27-38, 2007.

[14] M. Bierling, "Displacement estimation by hierarchical blockmatching," in Symposium on Visual Communications and Image Processing, vol. 1001 of Proceedings of SPIE, pp. 942-951, Cambridge, Mass, USA, 1988. 

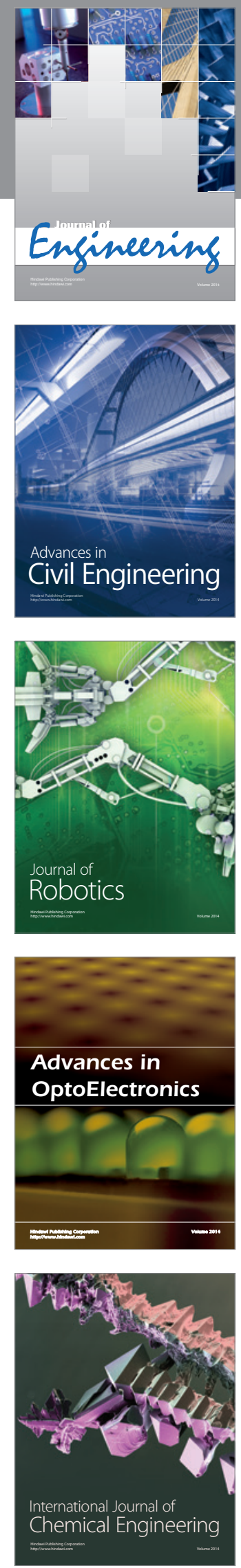

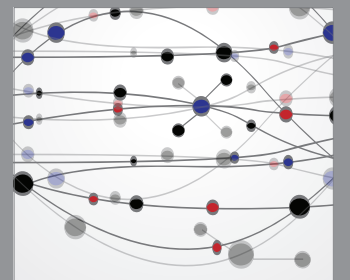

The Scientific World Journal
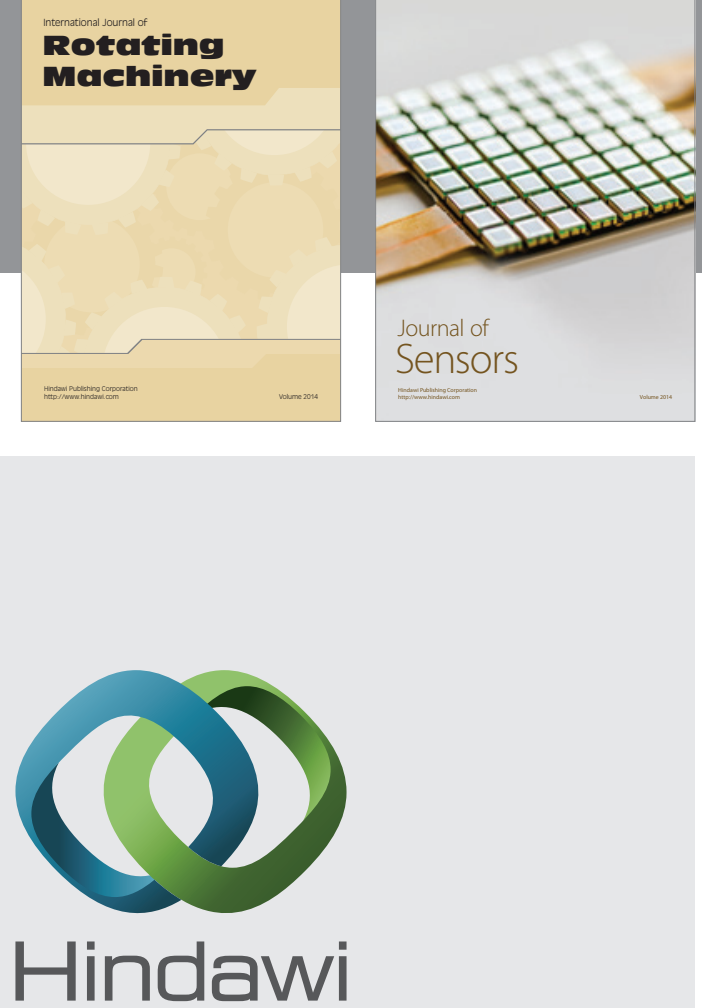

Submit your manuscripts at http://www.hindawi.com
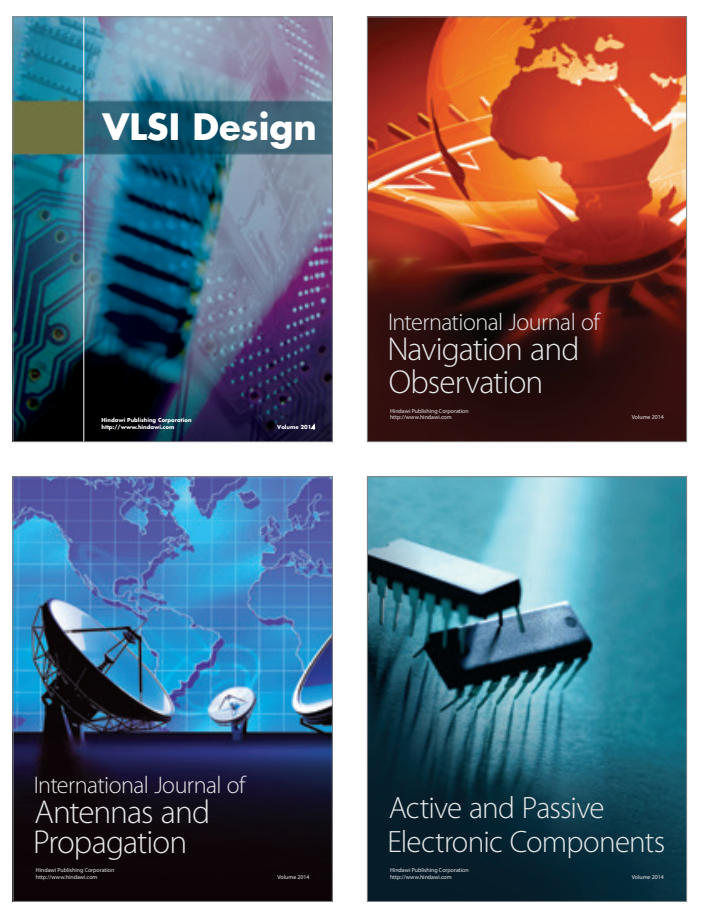
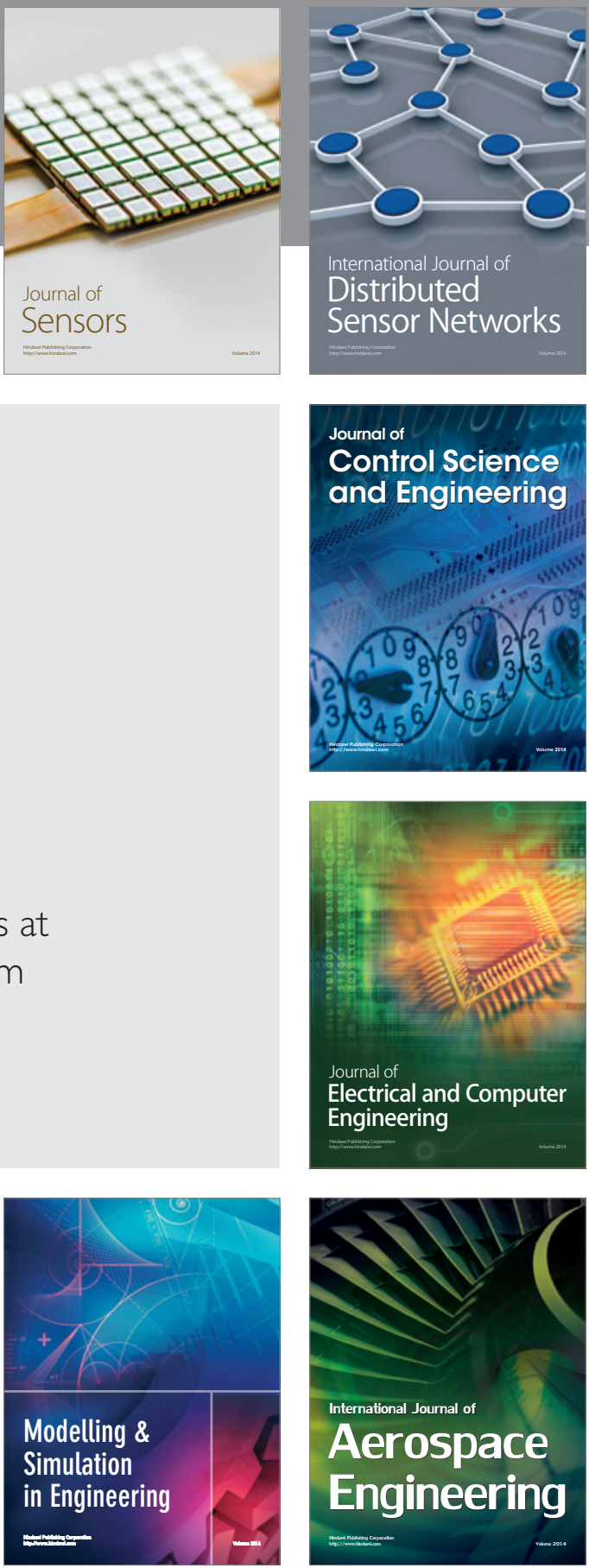

Journal of

Control Science

and Engineering
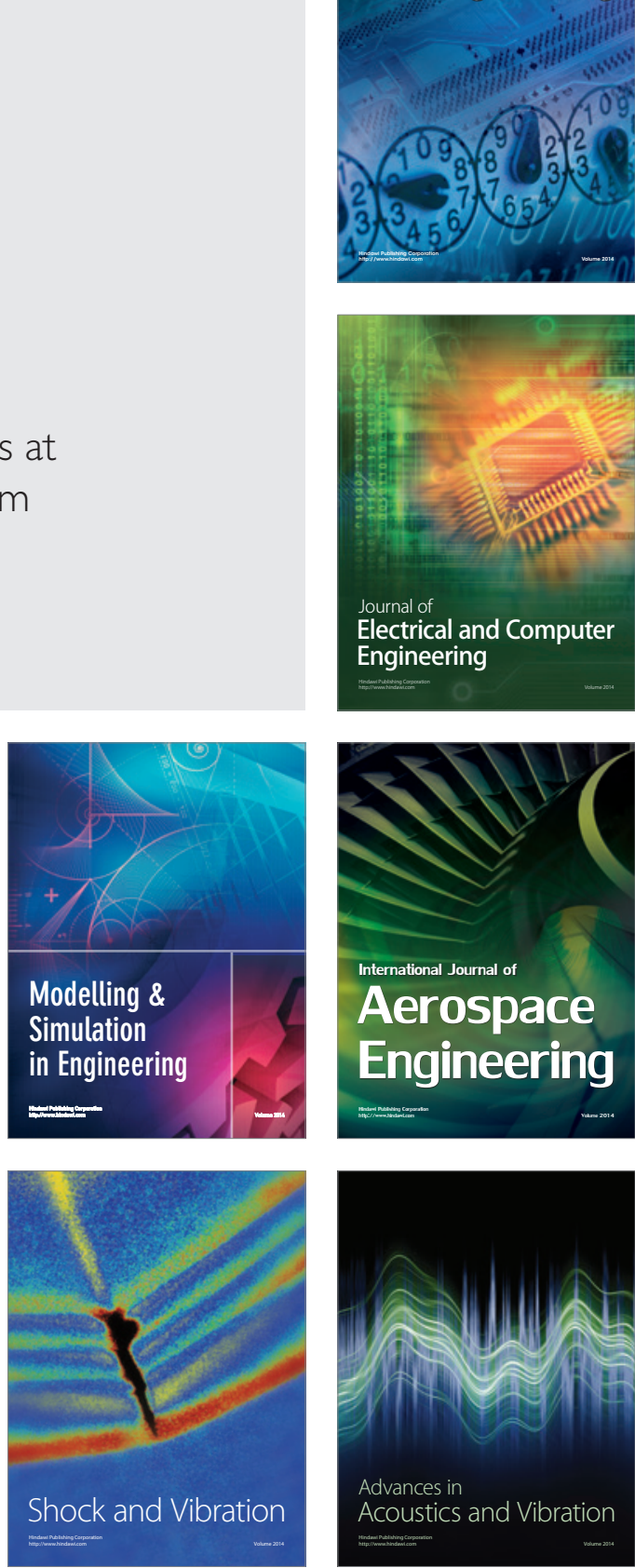